PROCEEDINGS OF THE

AMERICAN MATHEMATICAL SOCIETY

Volume 133, Number 8, Pages 2201-2205

S 0002-9939(05)07570-2

Article electronically published on March 4, 2005

\title{
A REDUCTION OF THE JACOBIAN CONJECTURE TO THE SYMMETRIC CASE
}

\author{
MICHIEL DE BONDT AND ARNO VAN DEN ESSEN
}

(Communicated by Bernd Ulrich)

\begin{abstract}
The main result of this paper asserts that it suffices to prove the Jacobian Conjecture for all polynomial maps of the form $x+H$, where $H$ is homogeneous (of degree 3) and $J H$ is nilpotent and symmetric. Also a 6dimensional counterexample is given to a dependence problem posed by de Bondt and van den Essen (2003).
\end{abstract}

\section{INTRODUCTION}

Let $F=\left(F_{1}, \ldots, F_{n}\right): \mathbb{C}^{n} \rightarrow \mathbb{C}^{n}$ be a polynomial map, i.e. each $F_{i}$ is a polynomial in $n$ variables over $\mathbb{C}$, and denote by $J F:=\left(\frac{\partial F_{i}}{\partial x_{j}}\right)_{1 \leq i, j \leq n}$ the Jacobian matrix of $F$. Then the Jacobian Conjecture asserts that if $\operatorname{det} J F \in \mathbb{C}^{*}$, then $F$ is invertible. It was shown in the classical papers [1] and [13] by Bass-Connell-Wright and Yagzhev, respectively, that it suffices to prove the Jacobian Conjecture for all $n \geq 2$ and all polynomial maps of the form $F=x+H$, where $H$ is homogeneous (of degree 3 ) and $J H$ is nilpotent.

In [12] and [7] the cubic homogeneous cases in dimension 3 (resp. 4) were treated by Wright (resp. Hubbers).

Recently, in [6] Washburn and the second author treated one more special case, namely they showed that if $n \leq 4$, then the Jacobian Conjecture holds for all polynomial maps of the form $F=x+H$, where $J H$ is homogeneous, nilpotent and symmetric.

At first glance the condition that $J H$ is symmetric seems rather special. However the main result of this paper, Theorem 1.1, asserts that it suffices to prove the Jacobian Conjecture for all $n \geq 2$ and all polynomial maps of the form $F=x+H$, where $J H$ is homogeneous, nilpotent and symmetric!

The technique to obtain this result is used in section 2 to give a negative answer in dimension 6 to a dependence problem posed in 2] (which, if true, would have implied the Jacobian Conjecture). We refer to section 2 for more details. Finally we would like to mention that in 3 the authors have obtained the following extensions of the results from [6]: the Jacobian Conjecture holds for all $F$ of the form $x+H$, where $J H$ is nilpotent and symmetric in the case $n \leq 4$ ( $H$ need not be homogeneous) and in the case $n=5$ when $H$ is homogeneous.

Received by the editors June 30, 2003.

2000 Mathematics Subject Classification. Primary 14R15, 14R10.

Key words and phrases. Jacobian Conjecture, Hessian Conjecture, dependence problems.

(C)2005 American Mathematical Society 


\section{REDUCTION TO SYMMETRIC MATRICES}

Throughout this paper we use the following notation:

$\mathbb{C}[x]:=\mathbb{C}\left[x_{1}, \ldots, x_{n}\right]$ is the polynomial ring in $n$ variables over $\mathbb{C}$ and $H:=$ $\left(H_{1}, \ldots, H_{n}\right): \mathbb{C}^{n} \rightarrow \mathbb{C}^{n}$ is a polynomial map. Its Jacobian matrix is denoted by $J H$. It follows from the Poincaré lemma (see for example [5], 1.3.53) that $J H$ is symmetric iff there exists $f \in \mathbb{C}[x]$ such that $H=\left(f_{x_{1}}, \ldots, f_{x_{n}}\right)$ or equivalently such that $J H=\left(\frac{\partial^{2} f}{\partial x_{i} \partial x_{j}}\right)$, the Hessian matrix of $f$. We denote this matrix by $h(f)$.

Observe that

$$
h(f)=J\left(f_{x_{1}}, \ldots, f_{x_{n}}\right) .
$$

For $A \in M_{n}(\mathbb{C})$ we put $f \circ A:=f(A x)$. It is well known that

$$
h(f \circ A)=A^{t} h(f)_{\mid A x} A .
$$

Now we introduce $n$ new variables $y_{1}, \ldots, y_{n}$ and to $H$ as above we associate the polynomial $f_{H} \in \mathbb{C}[x, y]$ defined by

(3) $f_{H}:=(-i) H_{1}\left(x_{1}+i y_{1}, \ldots, x_{n}+i y_{n}\right) y_{1}+\ldots+(-i) H_{n}\left(x_{1}+i y_{1}, \ldots, x_{n}+i y_{n}\right) y_{n}$.

So if $S$ is the (invertible) linear map given by

$$
S:=\left(x_{1}-i y_{1}, \ldots, x_{n}-i y_{n}, y_{1}, \ldots, y_{n}\right),
$$

then $g_{H}:=f_{H} \circ S=(-i) H_{1}(x) y_{1}+\ldots+(-i) H_{n}(x) y_{n}$.

One readily verifies that $h\left(g_{H}\right)$ is of the form

$$
h\left(g_{H}\right)=\left(\begin{array}{cc}
* & (-i)(J H)^{t} \\
(-i) J H & 0
\end{array}\right) .
$$

In order to formulate the main result of this paper we introduce

Hessian Conjecture $\mathbf{H C}(\mathbf{n})$. Let $f \in \mathbb{C}[x]$. If $h(f)$ is nilpotent, then $F:=$ $\left(x_{1}+f_{x_{1}}, \ldots, x_{n}+f_{x_{n}}\right)$ is invertible.

It follows from (1) that if the $n$-dimensional Jacobian Conjecture is true, then $\mathrm{HC}(\mathrm{n})$ is true as well. The surprising point is now

Theorem 1.1. The Jacobian Conjecture is equivalent to the Hessian Conjecture. More precisely, if $H C(2 n)$ holds, then $x+H$ is invertible for every $H: \mathbb{C}^{n} \rightarrow \mathbb{C}^{n}$ with JH nilpotent.

The proof of this result is based on the following lemma.

Lemma 1.2. Let $H=\left(H_{1}, \ldots, H_{n}\right): \mathbb{C}^{n} \rightarrow \mathbb{C}^{n}$ and let $f_{H} \in \mathbb{C}[x, y]$ be as defined in (3). Then $J H$ is nilpotent iff $h\left(f_{H}\right)$ is nilpotent.

Proof. Introduce an extra variable $z$ and write $f$ (resp. $g$ ) instead of $f_{H}$ (resp. $\left.g_{H}\right)$. Then $h(f)$ is nilpotent iff $\operatorname{det}\left(z I_{2 n}-h(f)\right)=z^{2 n}$. Put $q:=(1 / 2) \sum_{j=1}^{n}\left(x_{j}^{2}+y_{j}^{2}\right)$. Then $h(z q)=z I_{2 n}$, so

$$
h(z q-f)=z I_{2 n}-h(f) .
$$

Since det $S=1$, it follows from (2) and (5) that

$$
\operatorname{det} h(z q \circ S-g)=\operatorname{det} h(z q-f)_{\mid S(x, y)} .
$$


Since $q \circ S=\frac{1}{2} \sum_{j=1}^{n} x_{j}^{2}-\sum_{j=1}^{n} i x_{j} y_{j}$, it follows from (4) that

$$
h(z q \circ S-g)=\left(\begin{array}{cc}
* & -i z I_{n}+i(J H)^{t} \\
-i z I_{n}+i J H & 0
\end{array}\right) .
$$

Consequently

$$
\operatorname{det} h(z q \circ S-g)=\operatorname{det}\left(z I_{n}-J H\right) \operatorname{det}\left(z I_{n}-(J H)^{t}\right) .
$$

So by (6) and (7) we obtain

$$
\operatorname{det}\left(z I_{2 n}-h(f)\right)_{\mid S(x, y)}=\operatorname{det}\left(z I_{n}-J H\right) \operatorname{det}\left(z I_{n}-(J H)^{t}\right) .
$$

Hence $h(f)$ is nilpotent iff $\operatorname{det}\left(z I_{2 n}-h(f)\right)=z^{2 n}$ iff $\operatorname{det}\left(z I_{n}-J H\right)=z^{n}$ iff $J H$ is nilpotent.

Proof of Theorem 1.1. Let $H=\left(H_{1}, \ldots, H_{n}\right)$ be such that $J H$ is nilpotent and let $f_{H}$ be as in (3). Then by Lemma $1.2 h(f)$ is nilpotent. So the assumption $\mathrm{HC}(2 \mathrm{n})$ implies that $F=\left(x_{1}+f_{x_{1}}, \ldots, x_{n}+f_{x_{n}}, y_{1}+f_{y_{1}}, \ldots, y_{n}+f_{y_{n}}\right)$ is invertible. Consequently $F \circ S$ is invertible. An easy calculation shows that

$$
\begin{aligned}
F \circ S= & \left(x_{1}-i y_{1}-i \sum_{j} H_{j x_{1}}(x) y_{j}, \ldots, x_{n}-i y_{n}-i \sum_{j} H_{j x_{n}}(x) y_{j},\right. \\
& \left.y_{1}+\sum_{j} H_{j x_{1}}(x) y_{j}-i H_{1}, \ldots, y_{n}+\sum_{j} H_{j x_{n}}(x) y_{j}-i H_{n}\right) .
\end{aligned}
$$

Hence $S^{-1} \circ F \circ S=\left(x_{1}+H_{1}(x), \ldots, x_{n}+H_{n}(x), *, \ldots, *\right)$ is invertible, which in turn implies that $x+H$ is invertible.

Corollary 1.3. It suffices to prove the Jacobian Conjecture for all $n \geq 2$ and all $F$ of the form $F=\left(x_{1}+f_{x_{1}}, \ldots, x_{n}+f_{x_{n}}\right)$, where $h(f)$ is nilpotent and $f$ is homogeneous of degree 4 (or equivalently for all $n \geq 2$ and all $F$ of the form $F=x+H$ with $J H$ nilpotent and symmetric and $H$ homogeneous of degree 3 ).

Proof. Follows immediately from Theorem 1.1 and Corollary 2.2 of [1].

\section{Dependence PRoblems}

In the search for the Jacobian Conjecture the following problems were formulated by several authors (see [8], Conjecture 1, p. 80, [10], Conjecture B, p. 135, 11, Conjecture 11.3, 4] and [5], 7.1.7)

(Homogeneous) Dependence Problem (H)DP(n). Let $H:=\left(H_{1}, \ldots, H_{n}\right)$ with $H(0)=0$ be (homogeneous of degree $d \geq 1$ ) such that $J H$ is nilpotent. Are the $H_{i}$ linearly dependent over $\mathbb{C}$ ?

One easily verifies that the linear dependence of the $H_{i}$ is equivalent to the linear dependence of the rows of $J H$ over $\mathbb{C}$. It is shown in [5], Theorem 7.1.7, that $\operatorname{DP}(2)$ has an affirmative answer and that for each $n \geq 3$ there are counterexamples. The easiest such example is the following:

$$
H_{1}=x_{2}-x_{1}^{2}, \quad H_{2}=x_{3}+2 x_{1}\left(x_{2}-x_{1}^{2}\right), \quad H_{3}=-\left(x_{2}-x_{1}^{2}\right)^{2} .
$$

The homogeneous dependence problem is still open; but in the cases $n=3, d=3$ and $n=4, d=3$, affirmative answers were obtained by Wright in [12] and Hubbers 
in [7]. Recently in [2] the corresponding dependence problems were formulated for Hessian matrices, i.e.

(Homogeneous) Symmetric Dependence Problem (H)SDP(n). Let $H$ with $H(0)=0$ be (homogeneous of degree $d \geq 1$ ) such that $J H$ is nilpotent and symmetric. Are the $H_{i}$ linearly dependent over $\mathbb{C}$ ?

The importance of these problems becomes clear if one combines Theorem 1.1 with the following result of [2].

Theorem 2.1 ([2, Theorem 2.1]). i) If $S D P(p)$ has an affirmative answer for all $p \leq n$, then $H C(n)$ holds.

ii) If $S D P(p)$ has an affirmative answer for all $p \leq n-2$ and $H S D P(p)$ for $p=n-1$ and $p=n$, then $H C(n)$ holds for all homogeneous $f \in \mathbb{C}[x]$.

The aim of this section is to relate the dependence problems stated before with the symmetric dependence problems. As a consequence we obtain a negative answer to $\operatorname{SDP}(6)$. More precisely

Example. Let $H=\left(H_{1}, H_{2}, H_{3}\right)$ be as in (8). Then $J H$ is nilpotent and $H_{1}, H_{2}, H_{3}$ are linearly independent over $\mathbb{C}$. Now let $f_{H}$ be as in (3). Then it follows from the next result and the fact that $\operatorname{DP}(2)$ holds, that $h\left(f_{H}\right)$ is a counterexample to $\operatorname{SDP}(6)$.

Proposition 2.2. If $n$ is minimal such that $(H) D P(n)$ does not hold, then (H)SDP(2n) does not hold either.

Proof. i) Suppose $(\mathrm{H}) \mathrm{DP}(\mathrm{n})$ does not hold and $n$ is minimal with this property. Then there exists $H: \mathbb{C}^{n} \rightarrow \mathbb{C}^{n}$ with $H(0)=0$ such that $J H$ is nilpotent and the rows of $J H$ are independent over $\mathbb{C}$.

Claim. The columns of $J H$ are also independent over $\mathbb{C}$.

Namely, if the columns of $J H$ are dependent over $\mathbb{C}$, then there exists $0 \neq v \in \mathbb{C}^{n}$ with $J H \cdot v=0$. Let $T \in G l_{n}(\mathbb{C})$ be such that its last column equals $v$. Then the last column of $J H \cdot T$ equals zero. So if we put $\tilde{H}:=T^{-1} \circ H \circ T$, then $J \tilde{H}=T^{-1} J H(T x) T$ is nilpotent and also its last column equals zero. In particular $\tilde{H}_{1}, \ldots, \tilde{H_{n-1}} \in \mathbb{C}\left[x_{1}, \ldots, x_{n-1}\right]$. Finally put $H_{*}:=\left(\tilde{H}_{1}, \ldots, \tilde{H_{n-1}}\right)$. Since the last column of $J \tilde{H}$ is zero, it follows readily that $J H_{*}$ is nilpotent and that the rows of $J H_{*}$ are linearly independent over $\mathbb{C}$ (since the rows of $J \tilde{H}$ are because those of $J H$ are by hypothesis). So $H_{*}$ contradicts the minimality of $n$.

ii) Therefore, the colums of $J H$ are independent over $\mathbb{C}$. Let $g_{H}$ and $f_{H}$ be as above. Then $h\left(g_{H}\right)$ has the form (4).

Claim. The rows $R_{j}$ of $h\left(g_{H}\right)$ are independent over $\mathbb{C}$ : namely suppose that $\sum_{j=1}^{2 n}$ $c_{j} R_{j}=0$ for some $c_{j} \in \mathbb{C}$. Since the rows of $(-i)(J H)^{t}$ are independent over $\mathbb{C}$ (since the columns of $J H$ are by i)), the zero matrix in the right corner of $h\left(g_{H}\right)$ in (4) implies that $c_{1}=\ldots=c_{n}=0$. So $\sum_{j=n+1}^{2 n} c_{j} R_{j}=0$. However the rows of $(-i) J H$ are also independent over $\mathbb{C}$ (by hypothesis), so also $c_{j}=0$ if $j>n$, which proves the claim.

iii) Finally, since $f_{H}=g_{H} \circ T\left(T:=S^{-1}\right)$ it follows from (2) that $h\left(f_{H}\right)=$ $T^{t} h\left(g_{H}\right)_{\mid T(x, y)} T$. Therefore, the rows of $h\left(f_{H}\right)$ are also independent over $\mathbb{C}$, which concludes the proof. 


\section{FinAl REMARKS}

Almost three months after this paper was submitted, the authors were notified by David Wright that the paper [9] by Guowu Meng had appeared on the internet, in which he obtained a result similar to ours. He also formulates a Hessian Conjecture and shows that the Jacobian Conjecture is equivalent to his Hessian Conjecture. Meng's Hessian Conjecture states that the Jacobian Conjecture holds for all gradient maps $\nabla f:=\left(f_{x_{1}}, \ldots, f_{x_{n}}\right)$. The difference between our Hessian Conjecture and the one formulated by Meng is that he considers all polynomial maps of the form $\nabla f$ with $\operatorname{det} h(f) \in \mathbb{C}^{*}$, where we only need to consider all polynomial maps of the form $x+\nabla f$, with $h(f)$ nilpotent. So our reduction is more refined in the sense that it preserves the nilpotency as formulated in the classical reduction theorems of [1] and [13.

\section{ADDED IN PROOF}

In a recent paper the authors gave an affirmative answer to $\operatorname{HDP}(3)$. Also, the first author found counterexamples to $\operatorname{HDP}(\mathrm{n})$ for all $n \geq 5$.

\section{REFERENCES}

[1] H. Bass, E. Connell and D. Wright, The Jacobian Conjecture: Reduction of degree and formal Expansion of the Inverse, Bulletin of the AMS, 7 (1982), 287-330. MR0663785 (83k:14028)

[2] M. de Bondt and A. van den Essen, Nilpotent symmetric Jacobian matrices and the Jacobian Conjecture, J. Pure Appl. Algebra, 193 (2004), no. 1-3, 61-70. MF2076378

[3] M. de Bondt and A. van den Essen, Nilpotent symmetric Jacobian matrices and the Jacobian Conjecture II, Pure Appl. Algebra, 196 (2005), 135-148.

[4] A.Cima, A, Gasull and F. Mañosas, The discrete Markus-Yamabe problem, Nonlinear Analysis: Theory, Methods \& Applications, 35 (1999), no. 3, 343-354. MR.1643454 (2000j:37030)

[5] A. van den Essen, Polynomial Automorphisms and the Jacobian Conjecture, Vol. 190, in Progress in Mathematics, Birkhäuser, 2000. MF,1790619 (2001j:14082)

[6] A. van den Essen and S. Washburn, The Jacobian Conjecture for symmetric matrices, J. Pure Appl. Algebra, 189 (2004), no. 1-3, 123-133. MR2038568 (2004m:14133)

[7] E. Hubbers, The Jacobian Conjecture: Cubic homogeneous maps in Dimension Four, Masters's thesis, University of Nijmegen, 1994.

[8] G. Meisters, Polyomorphisms conjugate to Dilatations, pp. 67-88 in Automorphisms of Affine Spaces, Kluwer Academic Publishers (ed. A. van den Essen), 1995. MR1352691 (97m:14020)

[9] G. Meng, Legendre Transform, Hessian Conjecture and Tree Formula, http://front.math.ucdavis.edu/math-ph/0308035.

[10] C. Olech, On the Markus-Yamabe stability conjecture, pp. 127-137 in Proc. of the Intern. Meeting on Ordinary Differential Equations and their Applications, University of Florence, 1995.

[11] K. Rusek, Polynomial Automorphisms, preprint 456, Inst. of Math. Polish Acad. of Sciences, IMPAN, Warsaw, 1989.

[12] D. Wright, The Jacobian Conjecture: linear triangularization for cubics in dimension three, Linear and Multilinear Algebra, 34 (1993), 85-97. MR.1334679|(96j:14008)

[13] A. Yagzhev, On Keller's problem, Siberian Math. J., 21 (1980), 747-754.

Department of Mathematics, Radboud University of Nijmegen, Postbus 9010, 6500 GL Nijmegen, The Netherlands

E-mail address: debondt@math.kun.nl

Department of Mathematics, Radboud University of Nijmegen, Postbus 9010, 6500 GL Nijmegen, The Netherlands

E-mail address: essen@math.kun.nl 